\title{
Caracterización físico química y sensorial de chocolate para taza, elaborado con harinas de quinua, maca y plátano
}

Physical-chemical and sensory characterization of chocolate for cup, made with quinoa, maca and banana
flours

\author{
Milagros S. Granda-Santos ${ }^{1}$ (D), Santos T. Leiva-Espinoza' ${ }^{1}$, Manuel Oliva ${ }^{1}$ (D), Manuel Emilio Milla Pino ${ }^{2}$ (D)
}

\section{RESUMEN}

La industria del chocolate contribuye a dinamizar la economía de miles de familias, de pequeños productores de cacao de todo el mundo. El objetivo de la investigación fue realizar la caracterización fisicoquímica y sensorial del chocolate para taza, elaborado con harinas de quinua, maca y plátano. Para ello, se inició con la producción y posterior análisis proximal de muestras para determinar el contenido de carbohidratos, cenizas, grasas, humedad, proteínas y energía total de cuatro formulaciones, a los que además se aplicó un análisis sensorial. Los resultados indicaron que la formulación T4 alcanzó mayor contenido de proteína con un valor de 13,18 (Nx6,25)g / 100g. siendo ligeramente superior al resto. Además, el chocolate para taza elaborado con $100 \%$ pasta de cacao, fue el que superó en grasa, humedad y energía total al resto de formulaciones. Por otro lado, en el análisis sensorial se determinó la existencia de diferencias estadísticas para aroma, sabor y textura en cada uno de las formulaciones evaluadas. Se concluye, que mediante la adición de harinas como la de quinua, maca y plátano, se mejora sustancialmente las características fisicoquímicas en cuanto a nivel de proteína, textura y carbohidratos del chocolate para taza, que además alcanzó niveles aceptables en el análisis sensorial.

Palabras claves: chocolate para taza, cacao, harina, nutrientes.

\begin{abstract}
The chocolate industry contributes to energize the economy of thousands of families, of small cocoa producers around the world. The objective of the research was to carry out the physicochemical and sensory characterization of chocolate for cup, elaborated with quinoa, maca and banana flours. For this purpose, it was started with the production and later proximal analysis of samples to determine the content of carbohydrates, ashes, fats, humidity, proteins and total energy of four formulations, to which it was also applied a sensory analysis. The results indicated that the T4 formulation reached a higher content of protein with a value of $13.18(\mathrm{Nx} 6.25) \mathrm{g} / 100 \mathrm{~g}$. being slightly higher than the rest. In addition, the cup chocolate made with $100 \%$ cocoa paste, was the one that exceeded the rest of the formulations in terms of fat, moisture and total energy. On the other hand, in the sensory analysis it was determined the existence of statistical differences for aroma, flavor and texture in each of the formulations evaluated. It is concluded that through the addition of flours such as quinoa, maca and banana, it is substantially improved the physicochemical characteristics as for protein level, texture and carbohydrates of drinking chocolate, which also reached acceptable levels in the sensory analysis.
\end{abstract}

Keywords: drinking chocolate, cocoa, flours, nutrients.

\footnotetext{
${ }^{1}$ Universidad Nacional Toribio Rodríguez de Mendoza de Amazonas, Instituto de Investigación para el Desarrollo Sustentable de Ceja de Selva, Chachapoyas, Perú.

${ }^{2}$ Universidad de Jaén, Facultad de Ingeniería Civil, Jaén, Perú.

*Autor de correspondencia.E-mail: sadith.granda.santos@gmail.com
} 


\section{I.INTRODUCCIÓN}

El chocolate forma parte de una industria multimillonaria, que contribuye al desarrollo económico de miles de familias de pequeños productores de cacao en todo el mundo (Pang Tahu, 2004). Este producto es descrito como una suspensión de partículas sin grasa (azúcar, sólidos de cacao y partículas de leche en polvo), dispersadas en manteca de cacao como una fase continua (Beckett, 2009; Sokmens y Gunes, 2006). Tiene como fuente de materia prima a las semillas o granos del cacao (Teobroma cacao L.), árbol que constituye un importante cultivo de las regiones tropicales y húmedas de todo el mundo (Cheesman, 1944).

El chocolate para taza, es sólo uno de los múltiples tipos de uso del cacao, y es producido por simple tueste de las semillas junto a procesos de transformación elemental, obteniendo la pasta de cacao que constituye una de las formas más básicas de este producto (INACAL, 2017b). Este tipo de chocolate, también se denomina chocolate negro, amargo o puro, y se sugiere que al formar una mezcla con al menos un $18 \%$ de harina o almidón, éste es considerado chocolate para taza, propiamente dicho (INACAL, 2017a).

Hoy en día, el mercado globalizado de productos como el chocolate para taza, lo constituyen consumidores cada vez más exigentes y sugieren la elaboración de productos innovadores que puedan satisfacer la múltiples necesidades de la alimentación humana. Por lo que un chocolate de este tipo, no solo deberá tener mejor aroma, textura, sabor o color, sino también un incremento en el nivel nutricional, para que se complemente una necesidad en la alimentación, que puede alcanzarse mediante la adición de harinas de alto contenido nutricional a la fórmula de preparación del chocolate para taza (Vásquez, 2009; Valenzuela, 2007). Además podría convertirse en una alternativa de producción y mejora de valor agregado, para el pequeño productor de cacao y chocolate.

Las harinas de productos como la quinua, la maca o el plátano, son importantes fuentes de carbohidratos, proteínas, vitaminas y varios nutrientes adicionales. Esto los convierte en productos con un potencial ideal, y una adecuada respuesta a la mezcla con la pasta de cacao, que busca lograr un incremento en el valor nutricional en el chocolate para taza, sin afectar las características sensoriales del mismo (Piacente et al., 2002; Menezes et al., 2011; Mujica et al., 2006).

En la presente investigación, se formulan tres tipos de chocolates para taza, mediante la sustitución parcial de la pasta de cacao por harinas de quinua (30\%), maca $(30 \%)$ y plátano $(30 \%)$. Se pretende contribuir e incrementar el contenido nutricional de un chocolate para taza, con estas harinas, sin que haya alteraciones sustanciales en sus propiedades sensoriales. Por ello se realizará la caracterización fisicoquímica y sensorial, con el fin de determinar la composición final de un producto novedoso y con características nutricionales importantes.

\section{MATERIALY MÉTODOS}

\section{Proceso productivo de los chocolates para taza}

Se establecieron 4 formulaciones chocolate para taza: La T1 correspondió a una formulación de 100\% de pasta de cacao; la T2 correspondió a una formulación de $70 \%$ de pasta de cacao y $30 \%$ harina de quinua; la $\mathrm{T} 3$ correspondió a una formulación de $70 \%$ de pasta de cacao y $30 \%$ harina de maca; y la T4 correspondió a una formulación de $70 \%$ de pasta de cacao y $30 \%$ harina de plátano.

En la producción de los chocolates para taza se utilizaron granos de cacao fino de aroma, provenientes de plantaciones de cacao de productores de la Cooperativa de Servicios Múltiples APROCAM, ubicada en la provincia de Bagua, Región Amazonas. El proceso inicial se realizó en los ambientes de la empresa, Agroindustria Eco Products Consulting Groups SAC, y posteriormente en el Laboratorio de Cacao del Instituto de Investigación para el Desarrollo Sustentable de Ceja de Selva (INDES-CES )de la Universidad Nacional Toribio Rodríguez de Mendoza de Amazonas (UNTRM). Las harinas de quinua, maca y plátano fueron adquiridas en el mercado central de la ciudad de Chachapoyas, y no pasaron por tratamiento previo a su adición en la mezcla con la pasta de cacao. Se separó 
mediante cuarteo, una muestra de cacao de $500 \mathrm{~g}$ con un $7 \%$ de humedad, posteriormente, se determinó el calibre del grano de $1,1 \mathrm{~g}$, el cual sugirió su condición normal. Seguidamente, defectos como granos múltiples, quebrados y pasillas, fueron separados de la muestra y al finalizar se procedió a determinar el $85 \%$ de grado de fermentación mediante la prueba de corte (SINEACE, 2017).

El tostado de los granos de cacao se realizó en un tostador rotatorio de la marca Maquiagro Perú, al $80 \%$ de su capacidad, el cual fue sometido a calentamiento y luego programado a una temperatura constante de $120^{\circ} \mathrm{C}$. El tostado de los granos de cacao se produjo una vez encontrado el sonido del "crack del tostado", suceso que se alcanzó en un tiempo aproximado de 30 min. Posteriormente, los granos tostados de cacao pasaron por una descascarilladora de la marca Maquiagro Perú, obteniéndose los nibs de cacao (granos tostados sin cáscara), los que seguidamente fueron pasados por un molino de martillos para obtener el licor de cacao (Codini et al., 2004; Herrera y Ospina, 2016).

En el Laboratorio de cacao, cada uno de los componentes (o ingredientes) que conforman las formulaciones fueron colocados en un refinador de rodillos de piedra, marca SS Premier modelo PG503, durante 6 horas a una temperatura de $50^{\circ} \mathrm{C}$ logrando una pasta de cacao de 22 micras. Posteriormente, la mezcla fue sometida a un proceso de templado manual, colocando el producto en un recipiente con baño maría logrando una fluctuación de temperatura desde $50^{\circ} \mathrm{C}$ (alta) hacia $27^{\circ} \mathrm{C}$ (baja) y finalmente se mantuvo a $30^{\circ} \mathrm{C}$, batiendo la mezcla de manera constante. Con ello se generó el brillo en la tableta de chocolate para taza. Seguidamente, se procedió con el pesado y moldeado del chocolate, para lo cual se empleó moldes de acero inoxidable, con capacidad para un peso uniforme de $100 \mathrm{~g}$ por barra de chocolate. Este proceso finalizó realizando una ligera vibración manual de los moldes, para liberar las burbujas de aire generadas en el moldeado, para luego ser colocadas en un refrigerador a $16^{\circ} \mathrm{C}$ y por un tiempo aproximado de 15 min. Finalmente se empacó en papel manteca, y fue almacenado a temperatura ambiente hasta un posterior análisis fisicoquímico y sensorial.

Dentro del proceso de producción del chocolate, en cada una de las actividades fueron registrados los valores correspondientes a los pesos de entradas y pesos de salidas para estimar el balance de materia, lo cual permitió determinar pérdidas de producto a lo largo del proceso, verificando la eficiencia en cada una de las etapas.

\section{Caracterización fisicoquímica de los chocolates para taza}

Un total de $500 \mathrm{~g}$ de chocolate por cada formulación, fueron seleccionados y enviadas al laboratorio Sociedad de Tratamiento Técnico S.A.C. acreditado por el Instituto Nacional de Calidad (INACAL), a fin de determinar las características fisicoquímicas mediante un análisis proximal. Se determinó el contenido de carbohidratos, producto de la diferencia después de haber completado los análisis de cenizas, fibra, grasa total, proteína total y humedad; el contenido de cenizas, mediante la calcinación a temperaturas entre 550 $-600^{\circ} \mathrm{C}$ de la muestra; grasa, mediante el método de extracción Soxhlet; humedad, mediante secado en una estufa de $105^{\circ} \mathrm{C}-130^{\circ} \mathrm{C}$ hasta obtener un peso constante; proteínas, por el método Kjeldahl y finalmente la determinación de energía total por cálculo.

\section{Evaluación sensorial de los chocolates para taza}

Para la evaluación sensorial o nivel de aceptación de los chocolates para taza se emplearon a treinta panelistas sin entrenamiento, con el perfil del público consumidor, con un rango de edad de 18 a 30 años. Se preparó el chocolate haciendo una dilución de $100 \mathrm{~g}$ de cada formulación en un litro de agua hervida, por $5 \mathrm{~min}$. Este procedimiento se hizo para las cuatro formulaciones por separado. El chocolate en taza, se dejó enfriar por 3 min y se sirvió en vasos de 3 onzas previamente rotulados. Cada panelista recibió una pequeña instrucción en la que se indicó cómo se realizaría la prueba. Luego, cada uno degusto todas las formulaciones, para eso, se les hizo entrega de los cuatro tipos de muestras de chocolate, $60 \mathrm{ml}$ de cada una, con tres repeticiones 
distribuidas al azar. Se pidió a cada panelista que calificara la muestras de chocolate usando escalas hedónicas de 1, extremadamente desagradable, a 9, extremadamente agradable, para los atributos aroma, color, sabor y textura (Peryam y Pilgrim, 1957).

\section{Análisis de datos}

Para el análisis estadístico de la prueba organoléptica se utilizó la prueba de Friedman a un nivel de significación del 5\%, para identificar si existen diferencias significativas entre las fórmulas propuestas. Todo ello, fue procesado con el software estadístico Statistix 8.0.

\section{RESULTADOS}

Balance de materia del proceso productivo de los chocolates para taza

En la tabla 1 se presenta un resumen del balance de materia del proceso productivo (ingreso, salida y porcentaje de pérdida) de los chocolates para taza, donde se observa que el volumen de ingreso de granos de cacao fue de $3,40 \mathrm{~kg}$ para los ensayos de chocolate $100 \%$ pasta de cacao y de 2,40 kg para la obtención de chocolate con harinas de quinua, maca y plátano. Se observa también que a través del proceso de producción se generaron pérdidas, que variaron de acuerdo a cada etapa. Por ejemplo, en el descascarillado se produjo en mayor intensidad (8,5\%). En el resto de etapas, las pérdidas fueron motivadas por la misma naturaleza del proceso, llegando finalmente a obtener un peso de $2,90 \mathrm{~kg}$ para el chocolate $100 \%$ pasta de cacao y un peso de 2,93 kg para los chocolates con harinas. Finalmente se detalla que las pérdidas totales alcanzaron el $15,4 \%$ en ambos procesos.

Tabla 1. Balance de materia del proceso productivo del chocolate para taza $100 \%$ pasta de cacao

\begin{tabular}{|c|c|c|c|c|c|c|}
\hline \multirow{2}{*}{ Operación } & \multicolumn{3}{|c|}{$100 \%$ pasta de cacao } & \multicolumn{3}{|c|}{ Pasta de cacao $(70 \%)+$ harinas $(30 \%)$} \\
\hline & Ingreso (kg) & Salida (kg) & Pérdida (\%) & Ingreso (kg) & Salida (kg) & Pérdida (\%) \\
\hline Recepción del grano & 3,40 & 3,40 & 0,00 & 2,40 & 2,40 & 0,00 \\
\hline Selección & 3,40 & 3,39 & 0,40 & 2,40 & 2,39 & 0,40 \\
\hline Tostado & 3,39 & 3,28 & 3,00 & 2,39 & 2,32 & 3,00 \\
\hline Descascarillado & 3,28 & 3,01 & 8,50 & 2,32 & 2,12 & 8,50 \\
\hline Molido & 3,01 & 2,98 & 1,00 & 2,12 & 2,10 & 1,00 \\
\hline Formulación & - & - & - & 3,00 & 3,00 & 0,00 \\
\hline Refinado & 2,98 & 2,95 & 1,00 & 3,00 & 2,97 & 1,00 \\
\hline Temperado & 2,95 & 2,92 & 1,00 & 2,97 & 2,94 & 1,00 \\
\hline Moldeado & 2,92 & 2,90 & 0,50 & 2,94 & 2,93 & 0,50 \\
\hline Refrigerado & 2,90 & 2,90 & 0,00 & 2,93 & 2,93 & 0,00 \\
\hline Envasado y etiquetado & 2,90 & 2,90 & 0,00 & 2,93 & 2,93 & 0,00 \\
\hline Total & & & 15,40 & & & 15,40 \\
\hline
\end{tabular}

\section{Caracterización fisicoquímica de los chocolates para taza}

La caracterización fisicoquímica de los chocolates para taza elaborados en base a cacao y con harinas de quinua, maca y plátano, se muestra en la tabla 2.

Se encontró que en cuanto al nivel de carbohidratos, el mayor nivel alcanzado corresponde al chocolate ela- borado en base a la formulación de $70 \%$ pasta de cacao más 30\% harina de quinua, seguido por el chocolate elaborado a base de $70 \%$ pasta de cacao más $30 \%$ harina de plátano, y el menor valor de carbohidratos lo obtuvo la formulación con $100 \%$ pasta de cacao. Por otro lado, en cuanto a los niveles de ceniza, se encontró

Tabla 2. Características fisicoquímicas de los tratamientos de los chocolates para taza

\begin{tabular}{|c|c|c|c|c|}
\hline \multirow{3}{*}{ Características fisicoquímicas } & \multicolumn{4}{|c|}{ Tratamientos } \\
\hline & \multirow{2}{*}{$\begin{array}{c}\text { Pasta de } \\
\text { cacao: } 100 \%\end{array}$} & \multicolumn{3}{|c|}{ Pasta de cacao al $70 \%+$} \\
\hline & & $\begin{array}{c}\text { Harina de } \\
\text { quinua: } \mathbf{3 0 \%}\end{array}$ & $\begin{array}{c}\text { Harina de } \\
\text { maca: } \mathbf{3 0 \%}\end{array}$ & $\begin{array}{c}\text { Harina de } \\
\text { plátano: } \mathbf{3 0} \%\end{array}$ \\
\hline Carbohidratos (g/100g) & 31,14 & 38,02 & 36,54 & 37,54 \\
\hline Ceniza $(g / 100 g)$ & 3,41 & 3,20 & 1,24 & 1,14 \\
\hline Energía Total ( kcal/100g) & 608,15 & 570,78 & 580,04 & 595,08 \\
\hline $\operatorname{Grasa}(g / 100 g)$ & 48,27 & 40,98 & 42,74 & 43,74 \\
\hline Humedad (g/100g) & 4,89 & 5,33 & 1,55 & 1,35 \\
\hline Proteína $((\mathrm{Nx} 6,25) \mathrm{g} / 100 \mathrm{~g})$ & 12,29 & 12,47 & 12,31 & 13,18 \\
\hline
\end{tabular}

72 Rev. de investig. agroproducción sustentable 4(2): 69-77, 2020 ISSN: 2520-9760 
que la formulación con $100 \%$ pasta de cacao fue el que alcanzó el nivel más alto. Por lo contrario, los chocolates elaborados a base de $70 \%$ pasta de cacao más $30 \%$ de harina de plátano alcanzó el nivel de cenizas más bajo. Además, los chocolates producidos a base de $100 \%$ pasta de cacao, fueron los que alcanzaron los niveles más altos de energía total por encima de los alcanzados por los chocolates elaborados con harinas.

Con respecto al nivel de grasa, los resultados indican que el chocolate elaborado a base de $100 \%$ pasta de cacao fue el que alcanzó el mayor nivel, mientras que el chocolate a base de pasta de cacao y harina de quinua fue el que menos grasa obtuvo. Del mismo modo, el nivel de humedad tuvo mayor nivel en el chocolate elaborado a base de $70 \%$ pasta de cacao más $30 \%$ de harina de quinua, seguido por los chocolates elaborados a base de $100 \%$ pasta de cacao, $70 \%$ pasta de cacao más $30 \%$ harina de plátano y $70 \%$ pasta de cacao más $30 \%$ de harina de maca.

Finalmente, y en lo que corresponde al nivel de proteínas alcanzado por cada uno de las formulaciones, se encontró que fue el chocolate producido a base de $70 \%$ pasta de cacao y $30 \%$ harina de plátano, el que alcanzó el más alto nivel proteico, seguido del chocolate elaborado a base de pasta de cacao y $30 \%$ de harina de quinua. Los chocolates elaborados a base pasta de cacao $70 \%$ más harina de plátano $30 \%$, así como el chocolate elaborado a base de pasta de cacao al 100\% alcanzaron los niveles más bajos de proteína.

\section{Caracterización organoléptica de chocolates para} taza en base a cacao y adición de harinas de quinua, maca y plátano

En cuanto los resultados vinculados a los atributos sensoriales evaluados a los tratamientos en estudio, para el aroma, se encontró que la formulación que alcanzó el mayor promedio fue para la formulación T1, evidenciándose además la existencia de diferencias significativas a favor de estos resultados (Tabla 3 , Figura 1). El resto de formulaciones tuvieron un comportamiento similar para esta variable. En cuanto al atributo de color, no se encontraron diferencias significativas, por lo que los tratamientos no difieren el uno del otro (Tabla 3, Figura 1). Con respecto al sabor, se encontró que las formulaciones T1 y T3, fueron los que lograron un mejor promedio calificativo sensorial, ambos con 5,77, valores que, además, difieren estadísticamente del resto de tratamientos (Tabla 3, Figura 1). El menor nivel de calificación en cuanto al sabor, fue alcanzado por el tratamiento que incluye harina de plátano en su formulación.

Finalmente, para la textura se evidencia que formulación T3, fue la que obtuvo el mejor calificativo con 6,52 de promedio, valor estadísticamente diferente del resto según la prueba de Friedman (Tabla 3, Figura 1). Por otro lado, el chocolate con la formulación T4 alcanzó un promedio calificativo de 4,93, siendo la más rezagado en cuanto a la textura.

La prueba de Friedman estableció diferencia tanto en el aroma, como en el sabor y en la textura, destacando las formulaciones T1 y T3. En cuanto a color no existen diferencias significativas entre los tratamientos.

Tabla 3. Prueba de Friedman para la evaluación sensorial (color, sabor, aroma y textura) de los chocolates para taza en base a cacao y con sustitución parcial de harinas de quinua, maca o plátano

\begin{tabular}{lccc}
\hline \multirow{2}{*}{ Atributo } & \multicolumn{2}{c}{$\begin{array}{c}\text { Prueba de } \\
\text { Friedman }\end{array}$} & $\begin{array}{c}\text { Observación } \\
\text { (Tratamiento }\end{array}$ \\
\cline { 2 - 3 } & $\mathbf{X}^{\mathbf{2}}$ & $\begin{array}{c}\text { P- } \\
\text { valor }\end{array}$ & $>$ ) \\
\hline Aroma & $15,139^{* *}$ & 0,0017 & $\mathrm{~T} 1$ \\
Color & $3,787^{\mathrm{ns}}$ & 0,2853 & Iguales \\
Sabor & $16,346^{* *}$ & 0,0010 & $\mathrm{~T} 1, \mathrm{~T} 3$ \\
Textura & $14,552^{* *}$ & 0,0022 & $\mathrm{~T} 3$ \\
\hline
\end{tabular}

$\mathrm{p}>0,05$ : No significativo (ns)

$\mathrm{p}<0,05$ : Significativo $(*)$

$\mathrm{p}<0,01$ : Altamente significativo $(* *)$

\section{IV.DISCUSIÓN}

Para la elaboración de los chocolates para taza en base a cacao y con adición de harinas de quinua, maca y plátano, se realizaron los procesos correspondientes para su producción y se determinó mediante el balance de materia, que en el tratamiento de $100 \%$ pasta de cacao la pérdida fue de un $15,40 \%$ con respecto a la materia prima que ingresa al sistema de producción. Eeste valor se asemeja a los resultados encontrados por Cañoles (2015), quien encontró perdidas promedio de $15 \%$ durante el proceso de elaboración de chocolate. Sin embargo, los resultados están por debajo de 

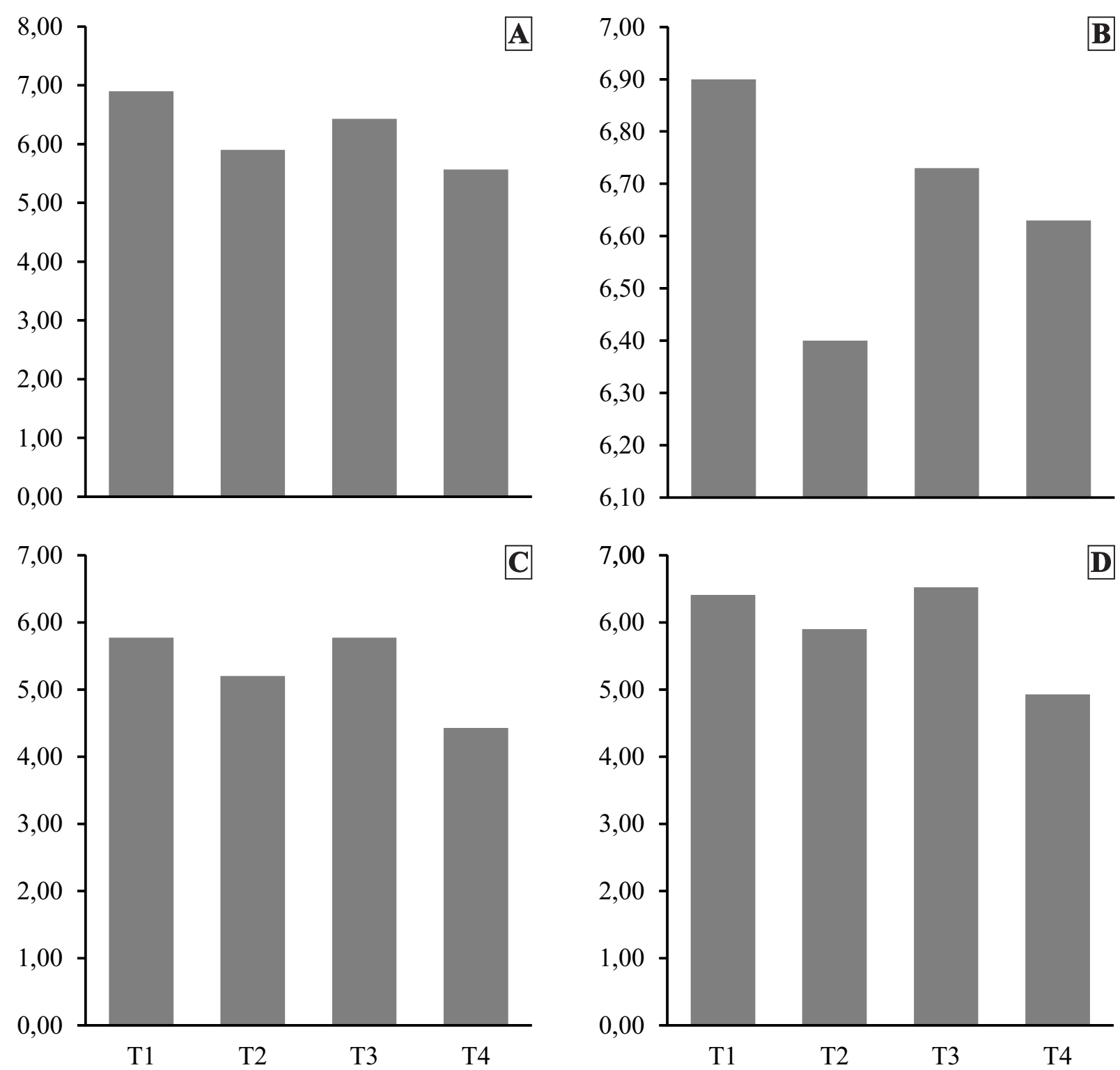

Figura 1. Valores promedio para los atributos sensoriales aplicados a los chocolates para taza formulados: A, aroma; B, color; C, sabor; D, textura.

los resultados de otros trabajos similares como los reportados por Jácome (2015), quien reportó que en el proceso de producción del chocolate se produjeron pérdidas por encima del $28 \%$.

En el balance de materia prima y con respecto a las formulaciones con adición de harinas, los resultados sugieren una tendencia similar a los alcanzados por la formulación T1, lo que podría indicar que las semillas de cacao con el cual se trabajó es de alta calidad y la sustitución de la pasta del cacao por el $30 \%$ de harinas, no representan ni determinan pérdidas mayores dentro del proceso de producción. Cabe señalar, que los mayores niveles de pérdida se produjeron en las etapas de descascarillado y tostado, resultados que se asemejan a los reportados en otros estudios como por ejemplo en el de Jácome (2015), donde refiere que sus pérdidas alcanzaron valores promedios de $18 \%$ para el descascarillado y $13 \%$ para el tostado. Sin embargo, la naturaleza del trabajo y realidades diferentes fueron determinantes y variaron en cuanto al porcentaje de perdida en la obtención de los resultados.

Por otro lado, y en referencia a la caracterización fisicoquímica que vincula al valor nutricional de las formulaciones, se determinó que en aquellas donde se utilizaron las harinas, se alcanzaron mayores niveles de carbohidratos que la formulación T1, indicando que 
los productos adicionados y utilizados como sustitutos parciales, contienen mayores niveles de carbohidratos que la pasta de chocolate. Lo contrario sucede cuando se evaluó el contenido de ceniza en las muestras, puesto que los mayores valores fueron encontrados en la pasta de cacao, siendo el chocolate formulado con harina de plátano el que tuvo niveles inferiores al 50\% de ceniza, menos que el tratamiento con pasta de cacao $100 \%$, existiendo una clara evidencia que la adición de harinas, disminuyen los niveles de ceniza en el chocolate para taza.

En referencia a la energía total, el chocolate con la formulación T1 fue el que alcanzó el mayor nivel, lo que permite suponer que el cacao contiene altos niveles de energía total. Sin embargo, cuando se adicionó o reemplazó con la harina de plátano, los valores no fueron significativamente inferiores, lo que tendría relación directa al alto contenido de carbohidratos presente en esta harina (Soto, 2010). Por otro lado, en el nivel de grasa, se aprecia un mayor nivel en la formulación T1, guardando relación con la naturaleza del producto, y es que el cacao tiene alto contenido de grasa, evidenciándose que fue superior al chocolate formulado con harinas (Canahua y Mujica, 2014).

Finalmente, para el contenido de proteína, se observó que el chocolate con la formulación T4 alcanzó el mayor nivel de proteína, guardando relación directa con la naturaleza del producto, pues ésta harina posee niveles de proteína sobre el 12,4\% (Canahua y Mujica, 2014). Además, se observó que fueron las formulaciones $\mathrm{T} 3$ y T2, respectivamente, las que alcanzaron los niveles de proteína más elevados, dejando rezagado en el último lugar al T1.

En relación a la caracterización organoléptica de chocolates para taza, se encontró que en referencia al aroma, el mayor nivel fue alcanzado por la formulación $\mathrm{T} 1$, lo que es lógico cuando se refiere a que la materia prima utilizada en su producción fue un cacao fino y caracterizado por poseer aromas exquisitos apreciables en el mundo chocolatero. Además, las formulaciones con harina, podría haberse disminuido los niveles de aroma. Estos resultados guardan rela- ción con el trabajo reportado por Portillo et al. (2009), donde destacan que los mayores niveles de aroma se encontraron en el tratamiento con mayor proporción de cacao.

Con respecto al color, los resultados no fueron significancia entre los valores, evidenciando que la adición de las harinas no lo modifica. Además, la contribución dominante de color que aporta la pasta de cacao en el proceso de producción del chocolate para taza es notoria. Definitivamente, estos resultados guardan una estructura lógica y relación directa con los encontrados por Schnermann y Schieberle (1997) donde hacen referencia que en una mezcla de chocolate enriquecido con otros agentes, variables como el aroma y color son dominantes en el producto final.

Por otro lado, el sabor tiene un comportamiento diferente, pues el chocolate más apetecible resultó ser el T1 y el T3. Posiblemente, los resultados guarden una estrecha relación con los compuestos volátiles que emite la maca en combinación con el chocolate. Resultados similares, fueron encontrados por Adaliht et al. (2016), cuando evaluaron la influencia de la adición de hierro y ácido linolénico de origen vegetal en el pan blanco, para su enriquecimiento y fortifica miento en base al requerimiento nutricional, utilizando como fuente la harina de maca, aportando sabor y textura a la masa de pan.

Finalmente, se encontró que los chocolates para taza que alcanzaron los mayores niveles de sabor, tuvieron los mejores niveles de textura, demostrando una relación directa entre estas dos variables. Algo semejante a lo reportado por Pérez (2018), quien comprobó que la adición de maca en trufas de chocolate tuvo diferencias significativas en cuanto a sabor y textura.

\section{CONCLUSIONES}

La caracterización fisicoquímica y sensorial de chocolate para taza, elaborado con harinas de quinua, maca y plátano sugieren que, mediante esta adicción, se produce una mejora sustancial del contenido de proteína, textura y carbohidratos. De esta manera, el producto resultaría más atractivo y beneficioso para los produc- 
tores y consumidores, puesto que, al incrementar el contenido nutricional del chocolate para taza, éste podría convertirse en un producto alternativo con alto potencial de desarrollo en el mercado del chocolate.

La adición de las harinas de quinua, maca y plátano no originó efecto negativo sobre las características sensoriales del chocolate para taza. Por lo contrario, éstas contribuyeron a obtener un producto tan apetecible como el chocolate elaborado $100 \%$ con pasta de cacao.

\section{REFERENCIAS BIBLIOGRÁFICAS}

Adaliht, J., A. Parillo, V. Choquehuanca, y V. Ibañez. 2016. "Efecto del enriquecido y fortificado con hierro y ácido linolénico en el pan blanco". Investigaciones Altoandinas 18 (2): 169 -178 .

Beckett S. 2008. The science of chocolate. Cambridge (Reino Unido): The Royal Society of Chemistry.

Canahua, A. y Á. Mujica. (2014). "Granos andinos: patrimonio y soporte de la seguridad alimentaria y nutricional sostenibles." Agroenfoque. (194), 44-46.

Cañoles, C. 2015. Evaluación de la línea de producción de figuras de chocolate en una empresa mediana de la Región de los Ríos. Tesis de Grado. Universidad Austral de Chile. Valdivia (Chile).

Cheesman, E. 1944. "Notes on the nomenclature, classification and possible relationships of cocoa populations". Trop Agricult, 21: $144-159$

Codini, M., F. Díaz, M. Ghirardi, I. Villavicencio. 2004. "Obtención y utilización de la manteca de cacao". Invenio. 7 (12): 143-148.

Herrera C. y N. Ospina. 2016. Nibs de cacao orgánico para mercados verdes. Tesis de Grado. Universidad Tecnológica de Pereira. Pereira (Colombia).

Jacome, W. 2015. Diseño de una planta de elaboración de chocolate negro y chocolate de leche a partir de licor de cacao. Tesis de Grado.
Escuela Politécnica Nacional. Quito (Ecuador).

Menezes, E. W., C. C. Tadini, T. B. Tribess, A. Zuleta, J. Binaghi, N. Pak, G. Vera, M. C. T. Dan, A. C. Bertolini, B. R. Cordenunsi, y F. M. Lajolo. 2011. "Chemical composition and nutritional value of unripe banana flour (Musa acuminata, var. Nanicão)." Plant Foods Hum Nutr 66: 231-237.

Mujica, A., R. Ortiz, A. Bonifacio, R. Saravia, G. Corredor, A. Romero, y S. Jacobsen. 2006. Agroindustria de la quinua (Chenopodium quinoa Willd.) en los países andinos. En Proyecto quinua: Cultivo multipropósito para los países andinos PNUD- PROY/INT/01/K01 Perú-Bolivia-Colombia. Informe Técnico. Lima (Perú).

INACAL (Instituto Nacional de Calidad). 2017a. "NTP-Codex Stan 87: Norma para el chocolate y los productos del chocolate". Lima (Perú).

INACAL (Instituto Nacional de Calidad). $2017 \mathrm{~b}$. "NTP-Codex Stan 141: Norma para la pasta de cacao (licor de cacao/chocolate) y torta de Cacao". Lima (Perú).

Pang Thau, J. 2004. "Rootstock effects on cocoa in Sabah, Malaysia”. Experimental Agriculture. 40 (4): 445-452.

Pérez, M. 2018. Maca andina: alimento funcional para la menopausia suplementado con calcio. Tesis de Grado. Universidad FASTA. Mar del Plata(Argentina).

Peryam, D. y F. Pilgrim. 1957. "Método de escala hedónica para medir las preferencias alimentarias". Tecnología de alimentos, 11:9-14.

Portillo E., M. Labarca, L. Grazziani, E. Cros, S. Assemat, F. Davrieux, R. Boulanger y M. Marcano. 2009. "Formación del aroma del cacao Criollo (Theobroma cacao L.) en función del tratamiento pos cosecha en Venezuela". Revista UDO Agrícola. 9 (2): 458-468.

Piacente S., V. Carbone, A. Plaza, A. Zampelli, y C. Pizza. 2002. "Investigación de los componen- 
tes del tubérculo de la maca (Lepidium meyenii Walp.)". J. Agr. Food Chem. 50: 56215625 DOI:10.1021/jf020280.

Schnermann, P. y P. Schieberle. 1997. "Evaluation of key odorants in milk chocolate and cocoa mass by aroma extract dilution analyses". $J$. Agric. Food Chem. 45 (3): 867-872.

SINEACE (Sistema Nacional de Evaluación, Acreditación y Certificación de la Calidad Educativa). 2017. "Normas de competencia para la post cosecha y la cata de licor o pasta de cacao". Lima (Perú).

Sokmen, A. y G. Gunes. 2006. "Influence of some bulk sweeteners on rheological properties of chocolate." LWT- Food Science and Technology 39 $\left(\begin{array}{ll}1 & 0\end{array}\right)$ : $1053-1058$. D O I : 10.1016/j.lwt.2006.03.002

Soto, V. 2010. "Cuantificación de almidón total y de almidón resistente en harina de plátano verde (Musa cavendishii) y banana verde (Musa paradisíaca)." Revista Boliviana de Química 27 (2): 94-99.

Valenzuela, A. 2007. "El chocolate un placer saludable." Revista chilena de nutrición 34 (3): 1-20. DOI: $10.4067 / \mathrm{S} 0717-75182007000300001$

Vásquez, E. 2009. Plan estratégico de Mercado para la promoción del consumo interno de derivados del cacao nacional. Informe final de consultoría. Lima (Perú): MINAGRI. 